\title{
Renoprotective Effect of Danhong Injection on Streptozotocin-Induced Diabetic Rats through a Peroxisome Proliferator-Activated Receptor $\gamma$ Mediated Pathway
}

\author{
Xue Yang $\mathbb{D}^{1,2}$ Xiang Xiao, ${ }^{1}$ Hailian Wang, ${ }^{3}$ Yi Li, $^{1}$ Li Wang, \\ Guisen Li $\mathbb{D})^{1,2}$ and Shaoping Deng $\mathbb{D}^{3}$ \\ ${ }^{1}$ Renal Division and Institute of Nephrology, Sichuan Academy of Medical Science and Sichuan Provincial People's Hospital, \\ School of Medicine, University of Electronic Science and Technology of China, Chengdu 610072, China \\ ${ }^{2}$ Southwest Medical University, Luzhou 646000, China \\ ${ }^{3}$ Institute of Organ Transplantation, Sichuan Academy of Medical Science and Sichuan Provincial People's Hospital, \\ Chengdu 610072, China
}

Correspondence should be addressed to Guisen Li; liguisen@med.uestc.edu.cn and Shaoping Deng; sdeng10@yahoo.com

Received 23 August 2017; Revised 10 December 2017; Accepted 11 January 2018; Published 11 April 2018

Academic Editor: Ki-Wan Oh

Copyright (C) 2018 Xue Yang et al. This is an open access article distributed under the Creative Commons Attribution License, which permits unrestricted use, distribution, and reproduction in any medium, provided the original work is properly cited.

\begin{abstract}
The aim of the study was to investigate the protective effect of Danhong injection (DHI) on diabetic kidney disease and explore the potential mechanisms. Diabetic kidney disease was induced by unilateral nephrectomy, high-fat diet, and streptozotocin. After DHI administration, the renal function deterioration, 24-hour total urine protein excretion, and elevated serum lipid levels were reversed to some extent, and the renal pathological damage was also ameliorated. The KEGG pathway enrichment analysis demonstrated that the PPAR $\gamma$ signal pathway was significantly upregulated in DH group. And the increased expressions of PPAR $\gamma$ and UCP-1 were confirmed by immunohistochemistry, whereas the p38MAPK was significantly decreased. These data show that DHI could delay the progress of DKD, and the effect might be achieved in part by activating the PPAR $\gamma$ signaling pathway.
\end{abstract}

\section{Introduction}

Diabetic kidney disease (DKD) is one of the diabetic microvascular complications characterized by proteinuria and progressively deterioration of renal function. Until today, a variety of treatment strategies still could not completely prevent the progress of DKD into end-stage renal disease (ESRD). Over the past decades, scientists have been trying to find different ways and agents to treat $\mathrm{DKD}$, but the results were not satisfactory [1-3].

For example, recently, a multinational prospective randomized controlled trial involving 8561 patients with type 2 diabetes mellitus and chronic kidney disease was prematurely terminated because of the severe adverse events of aliskiren, including increased risk of acute kidney injury, hyperkalemia, hypotension, and stroke [4]. Another study indicated that, among patients with type 2 diabetes mellitus and stage 4 chronic kidney disease, bardoxolone methyl, a nuclear factor
E2 related factor-2 activator, did not reduce the risk of ESRD or death from cardiovascular [5]. Moreover, DKD was still the leading cause of ESRD in the United States [6]. A recent report revealed that chronic kidney disease related to diabetes has become more common than chronic kidney disease related to glomerulonephritis in China [7]. Therefore, more effective drugs that can delay or prevent the progress of DKD are necessary.

Danhong injection (DHI) was extracted from Salvia miltiorrhiza and Carthami tinctorii and used widely for the treatment of cardiovascular diseases in China. Qualitative and quantitative characterization of DHI was effectively evaluated by high-performance liquid chromatography with diode array detection (HPLC-DAD) $[8,9]$. Previous studies indicated that DHI could decrease the levels of urinary albumin excretion rate (UAER) and 24-hour urinary protein in patients with DKD $[10,11]$. In experimental DKD rats, renal hypertrophy and $24 \mathrm{~h}$ urinary protein excretion were 
ameliorated by Tanshinone IIA, an active ingredient of Salvia miltiorrhiza [12]. Liu et al. reported that DHI inhibited the development of diabetic retinopathy and nephropathy in diabetic $\mathrm{db} / \mathrm{db}$ mice [13]. These data suggested that DHI could effectively delay the progress of DKD, but the molecular mechanisms for the renoprotective effects of DHI were still unclear. In this study, we established a DKD rat model to explore the renoprotective effect of DHI and investigate the underlying mechanisms.

\section{Materials and Methods}

2.1. Reagents. Rabbit anti-PPAR $\gamma$ and anti-UCP-1 polyclonal antibodies were purchased from Proteintech Group (Chicago, IL, USA). Rabbit anti-P38MAPK polyclonal antibody was obtained from Cell Signaling Technologies (Danvers, MA, USA). Streptozotocin (STZ) was purchased from Sigma-Aldrich (St. Louis, MO, USA).

2.2. Quality Control of DHI. Danhong injection was purchased from Heze Buchang Pharmaceutical Co., Ltd. (Shandong, China) (drug approval number: Z20026866). According to the criteria of China Food and Drug Administration, the quality control standard of DHI was that the total amounts of danshensu (molecular formula: $\mathrm{C}_{9} \mathrm{H}_{10} \mathrm{O}_{5}$ ) and protocatechuic aldehyde (molecular formula: $\mathrm{C}_{7} \mathrm{H}_{6} \mathrm{O}_{3}$ ) should not be lower than $0.5 \mathrm{mg}$ in $1 \mathrm{~mL}$ injection analyzed by high-performance liquid chromatography (HPLC). Meanwhile, the total flavonoids detected by visible spectrophotometry should not be lower than $5.0 \mathrm{mg} / \mathrm{mL}$ against rutin (molecular formula: $\mathrm{C}_{27} \mathrm{H}_{30} \mathrm{O}_{16}$ ) [14].

2.3. Animals. Male Sprague-Dawley rats (4 to 5 weeks) weighing 200-250 g were obtained from Dashuo Biotechnology Co., Ltd. (Chengdu, China). Rats were kept in a room with $12 \mathrm{~h}$ light-dark cycle (temperature $18-29^{\circ} \mathrm{C}$ and relative humidity $40-70 \%$ ) and were free to get food and drink. We tried our best to reduce the number of animals and suffering during the experiments. The protocols for in vivo study with rats accorded with the Guide for the Care and Use of Laboratory Animals published by NIH, and all processes conformed to international guidelines on the ethical use of animals. This study was approved by Institutional Review Boards of the Sichuan Academy of Medical Sciences and Sichuan Provincial People's Hospital.

2.4. Animal Model. After a week of adaptive feeding, all animals were given unilateral nephrectomy and then fed with a high-fat diet for 4 weeks. Twelve rats were selected as the control group (NM group, $n=12$ ), and the remaining rats were fasted for 12 hours and then injected intraperitoneally with STZ (38 mg/kg). After 72 hours and 7 days, the blood was withdrawn from the tail vein to measure glucose levels. If the blood glucose levels in NM group were normal and in model group were more than $16.7 \mathrm{mmol} / \mathrm{L}^{-1}$, it is recognized as a successful model. Forty rats with diabetic kidney disease were randomly divided into two groups: DHI injected group (DH group, $n=20$ ) and saline injected group (NS group, $n=20)$. DH group was given Danhong injection intraperitoneally daily $(2 \mathrm{ml} / \mathrm{kg}$ ) for two weeks; NM group and NS group were given saline intraperitoneally daily $(2 \mathrm{ml} / \mathrm{kg})$ for two weeks.

\subsection{Determination of Renal Functions and Serum Lipid Levels.}

Four rats were randomly chosen in each group to collect $24 \mathrm{~h}$ urine in metabolic cages at the 2nd, 6th, and 10th week after drug administration. The urine samples were used to detect 24-hour urine total protein (24 h TP). After anesthetizing the selected rats, abdominal aorta blood samples were gathered to determine blood urea nitrogen (BUN), serum creatinine (Cr), cystatin C (Cys-C), total cholesterol (TC), triglyceride (TG), low-density lipoprotein cholesterol (LDL-C), and highdensity lipoprotein cholesterol (HDL-C) by automatic biochemical analyzer (Hitachi, Japan).

2.6. Identification of DEGs and KEGG Pathway by Enrichment Analysis. Total RNA from the kidneys in the three groups ( $n=3$ per group for each time point) were isolated by TRIzol ${ }^{\circledR}$ Reagent (Invitrogen Life Technologies), and then RNA was qualified and quantified by NanoDrop ND-1000. Samples were marked by Arraystar RNA Flash Labeling Kit and hybridization was performed by Agilent SureHyb. The hybridized arrays were washed, fixed, and scanned by using the Agilent DNA Microarray Scanner (part number G2505C). Agilent Feature Extraction software (v11.0.1.1) was used to capture the chip probe signal values. Agilent GeneSpring GX v12.1 software was used to standardize chip and select the differential expression mRNA (fold change $\geqslant 2.0$, $P$ value $\leqslant 0.05)$. Then the differentially expressed mRNA was analyzed based on Kyoto Encyclopedia of Gene and Genomes (KEGG) pathway.

2.7. Determination of Morphology and the Expression of PPAR $\gamma, U C P-1$, and P38MAPK in the Kidney. After the rats were sacrificed at different time points mentioned above, the right kidneys were removed and fixed in $4 \%$ paraformaldehyde followed by embedding in paraffin. To evaluate the renal pathological changes and the glomerular volume, the kidney $5 \mu \mathrm{m}$ cross paraffin sections were prepared and stained with periodic acid-Schiff (PAS). The slides were also used to detect the expression of PPAR $\gamma$, uncoupling protein-1 (UCP1), and p38MAPK by immunohistochemistry staining. Three sections were stained from each kidney and there were 4 rats in each group.

2.8. Statistical Analysis. Data were presented as mean $\pm \mathrm{SD}$ and the significant difference was considered at $P<0.05$. When the variance is homogeneous, use one-way ANOVA; if not, use rank sum test. Statistical analysis was performed using SPSS software (version 16.0; SPSS Inc., Chicago, IL).

\section{Results}

3.1. Effects of DHI on Renal Function. We examined the serum levels of BUN, Cr, Cys-C, and $24 \mathrm{~h}-\mathrm{TP}$ to determine the therapeutic effect of DHI for DKD rats. The levels of 

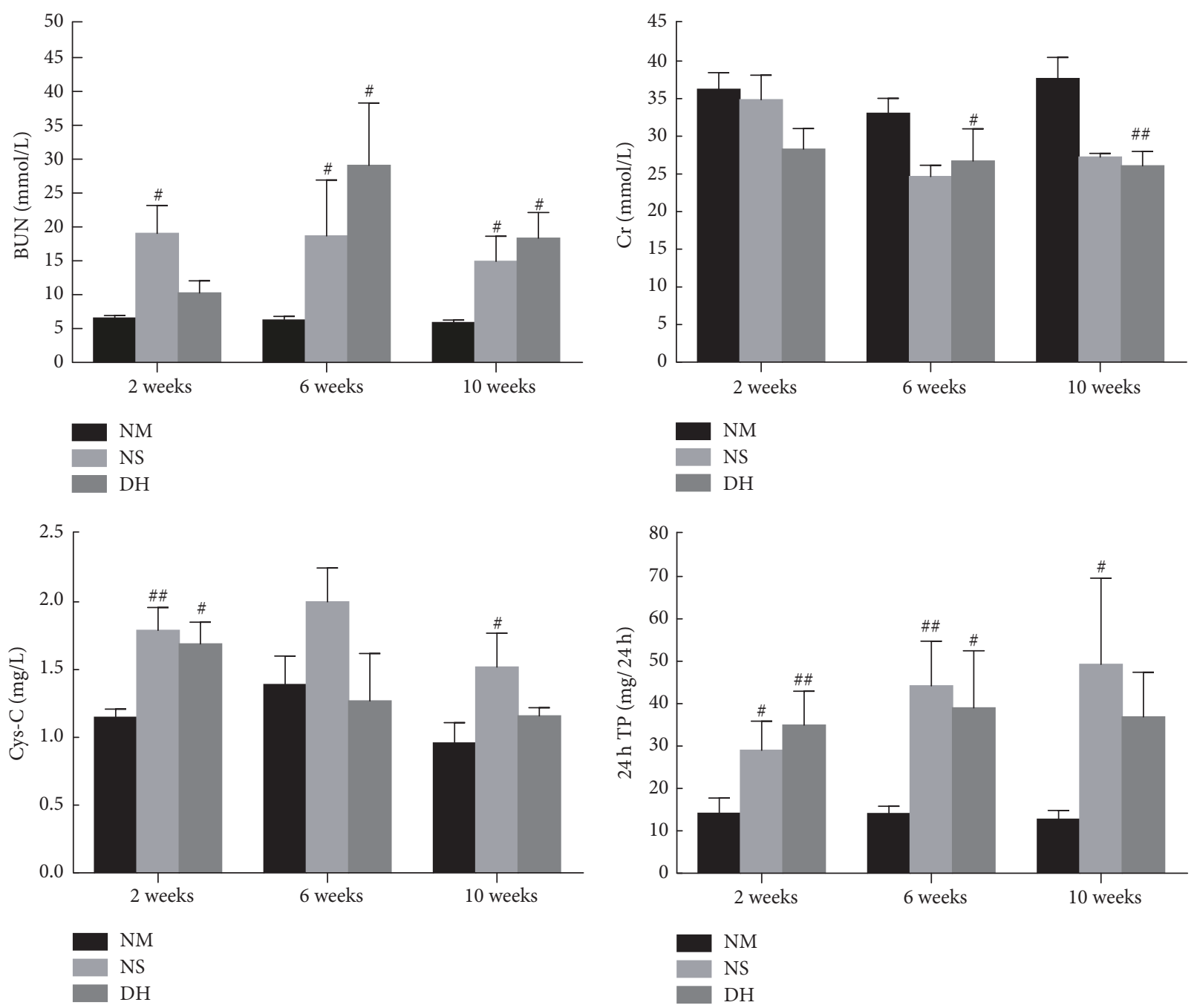

FIGURE 1: Effects of DHI on renal function and 24-hour urine total protein. The levels of BUN, Cr, Cys-C, and 24 h-TP were measured at the 2nd, 6th, and 10th week after administration by automatic biochemical analyzer. ${ }^{\#} P<0.05$ and ${ }^{\# \#} P<0.01$ versus the control group (NM).

BUN, Cys-C, and $24 \mathrm{~h}$ TP in the NS group were significantly higher than those in the NM group. After DHI intraperitoneal administration for 14 days, the changes of serum BUN and $\mathrm{Cr}$ showed a downward trend in $\mathrm{DH}$ group compared with NS group (Figure 1). Moreover, although the $24 \mathrm{~h}$ TP levels of rats in DH group were higher than NS group at the end of the 2nd week, Danhong injection decreased $24 \mathrm{~h}$ TP levels at the 6th and 10th week. In addition, the Cys-C levels of rats in the $\mathrm{DH}$ group were lower than that in the NS group at each time point after DHI administration.

3.2. Effects of DHI on Kidney Morphology. The characteristic pathological changes in DH group and NS group included increased glomerular volume, mesangial matrix proliferation, increased mesangial cells, and thickening basal membrane. Comparing with NS group, the DH group showed alleviated pathological damage at each time point after DHI administration (Figure 2(a)). As showed in Figure 2(b), there was no significant difference about the glomerular volume among each group at the 2nd week. But at the 6th and 10th week, glomerular volume in the $\mathrm{DH}$ group was smaller than that of the NS group, especially at the 6th week $(P<0.05)$.

3.3. Effects of DHI on Serum Lipid Levels. To assess the circulating lipid profiles, the serum samples were collected to detect the levels of TC, TG, LDL-C, and HDL-C. The results indicated that DHI significantly reduced TC and LDLC levels $(P<0.05)$ and elevated HDL-C $(P<0.01)$ levels in diabetic kidney disease rats at the 10th week after treatment. Meanwhile, the level of TC in DH group was lower than that of the NS group at the $2 \mathrm{nd}$ and 6 th week. However, the serum of TG was not affected significantly (Figure 3 ).

3.4. DEGs Selection and KEGG Pathway Enrichment Analysis. The microarray assay identified a number of mRNAs that were expressed differentially between DH group and NS group. A total of 464 genes were screened out, including 185 upregulated genes and 279 downregulated genes. The hierarchical clustering of differentially expressed genes of the 

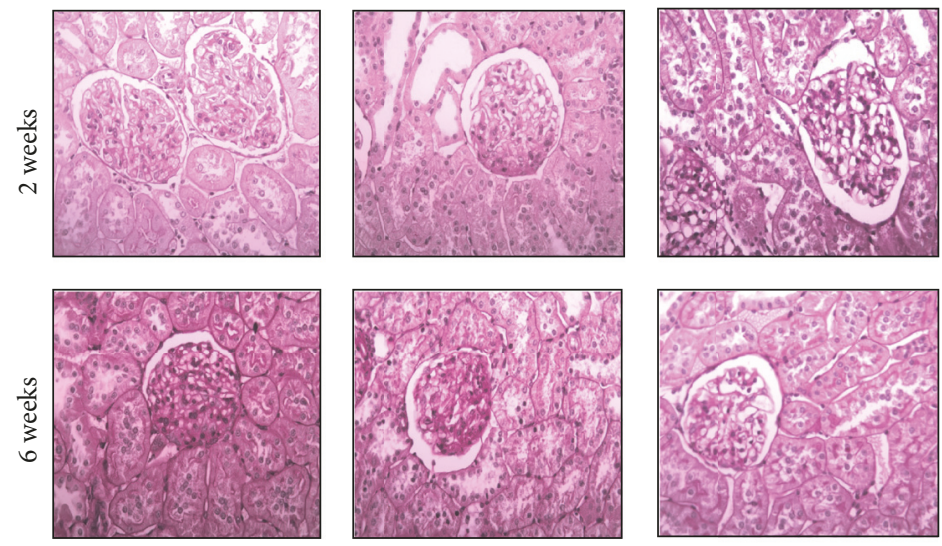

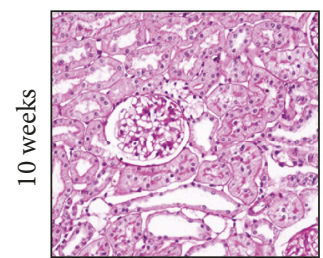

NM

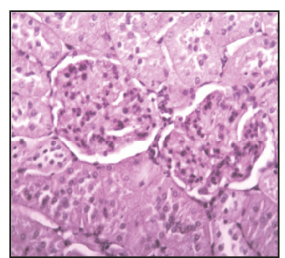

NS

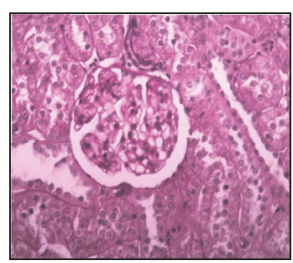

$\mathrm{DH}$

(a)

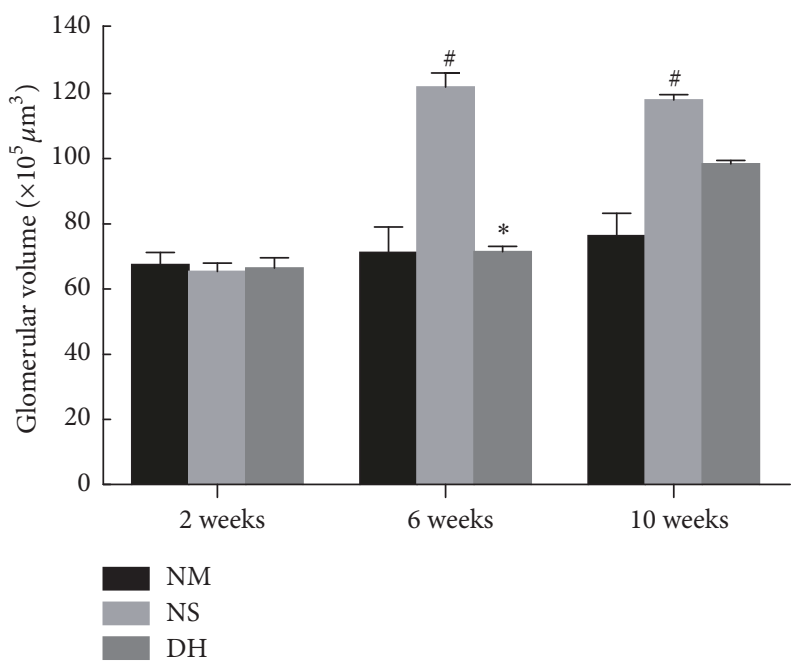

(b)

FIGURE 2: Effects of DHI on renal morphology and glomerular volume. After the rats were sacrificed at the 2nd, 6th, and 10th week, the kidney $5 \mu \mathrm{m}$ cross paraffin sections were prepared and stained with PAS (a) (scale bar: $500 \mu \mathrm{m}$ ), and the glomerular volumes were quantified (b). ${ }^{\#} P<0.05$ versus the control group (NM); ${ }^{*} P<0.05$ versus the saline injection group (NS).

three groups was performed and the results were showed in Figure 4(a). The NM group and DH group were similar and they were well separated from the NS group. The KEGG pathway enrichment analysis indicated that 34 pathways were revealed to be notably affected in the $\mathrm{DH}$ group. The top 10 pathways upregulated by DHI are displayed in Figure 4(b). The most significant biological pathway was PPAR signaling pathway enriched with 6 genes: acyl-CoA synthetase bubblegum family member 1 (ACSBG1), adiponectin (ADIPOQ), fatty acid binding protein 4 (FABP4), similar to fatty acid translocase/CD36 (RGD1565355), solute carrier family 27 member 5 (SLC27A5), and uncoupling protein-1 (UCP-1).
3.5. Effects of DHI on the Expression of PPAR $\gamma$, UCP-1, and p38MAPK in the Kidney. To test the effect of DHI on the PPAR $\gamma$ signaling pathway, we chose PPAR $\gamma$ and its downstream signaling molecule UCP-1, which were both involved in PPAR $\gamma$ signaling pathway, as well as p38MAPK, which was related to the PPAR $\gamma$ signaling pathway, for further validation. Immunohistopathologic staining for the three proteins was performed in rat kidney tissues. As shown in Figures 5(a) and 5(b), the reduced expression levels of PPAR $\gamma$ and UCP-1 were significantly restored after treatment with DHI. The staining of p38MAPK in NS group was much stronger than NM group (Figure 5(c)). 

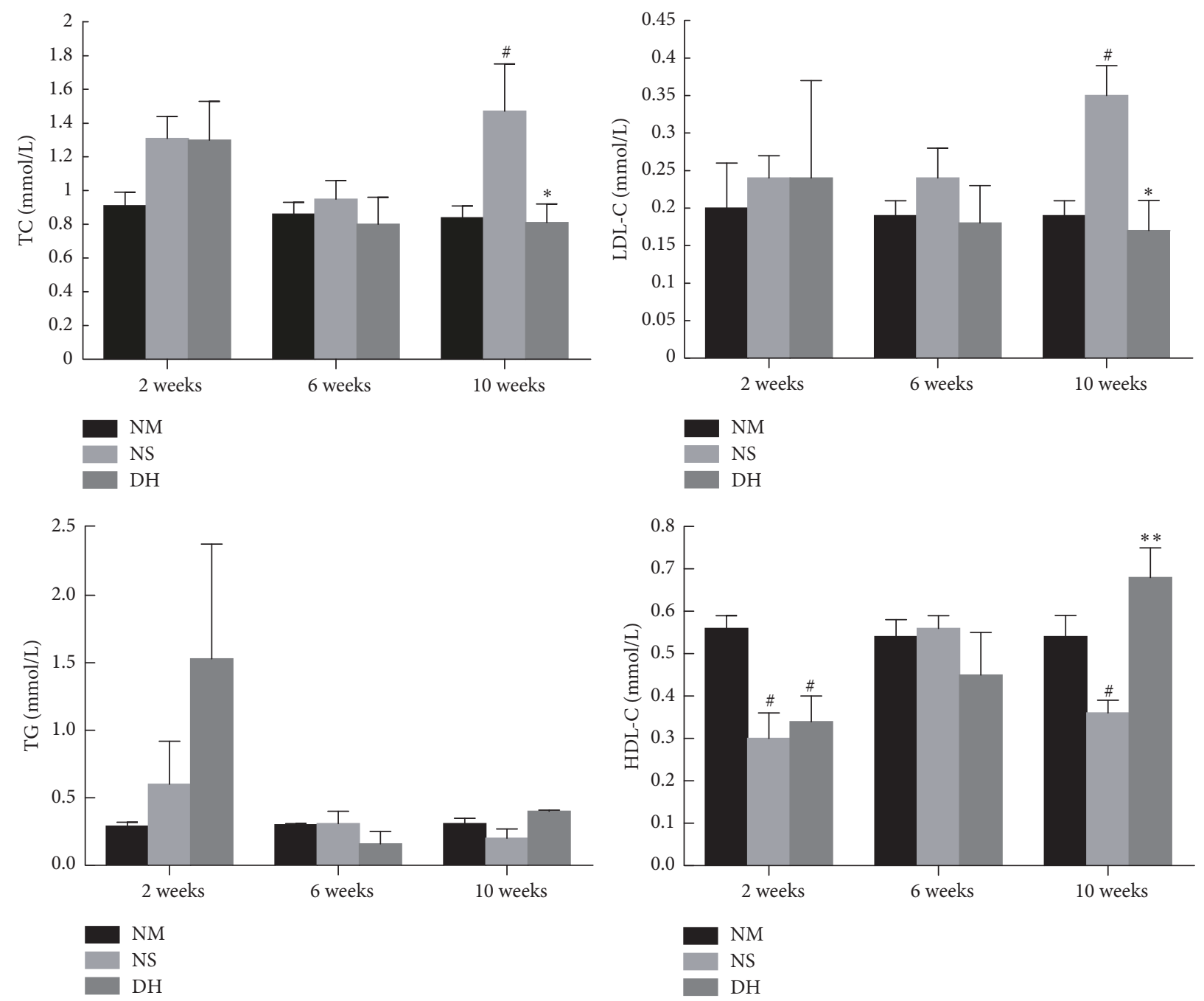

FIGURE 3: Effects of DHI on serum lipid levels. To test the circulating lipid profiles, the blood samples were collected at the 2nd, 6 th, and 10th week after administration. ${ }^{\#} P<0.05$ versus the control group $(\mathrm{NM}) ;{ }^{*} P<0.05$ and ${ }^{* *} P<0.01$ versus the saline injection group (NS).

\section{Discussion}

The main components of DHI are tanshinone, salvia acid, salvianolic acid, safflower yellow pigment, safflower phenolic glycosides, and catechol [15]. Due to its antioxidant, antiapoptotic, anti-inflammatory, antithrombotic and antifibrinolytic effects, Danhong injection alleviated myocardial ischemia/ reperfusion injury in rats [16] and minipigs [17], as well as cerebral ischemia/reperfusion injury in rats $[14,18]$, and exerted the protective effect in systemic acute inflammatory reaction [19], acute lung injury [20], and acute hepatic failure [21]. As for diabetic kidney disease, meta-analysis suggested that DHI can improve urinary albumin excretion rate (UAER) levels in patients $[22,23]$. However, the renoprotective mechanism of DHI for diabetic kidney disease has not yet been fully defined.

Our study demonstrated that, after DHI administration, the renal function deterioration, 24-hour total urine protein excretion, and elevated serum lipid levels were reversed to some extent, and the renal pathological damage was also ameliorated. All of these data indicated that DHI has the potential renoprotective effect on STZ induced diabetic kidney disease. Moreover, the KEGG pathway enrichment analysis indicated that these effects partially depended on the activation of PPAR $\gamma$ signaling pathway by DHI. We further validated the differential gene expression by immunohistochemistry staining. The results confirmed that the PPAR $\gamma$ and UCP-1 were significantly decreased in kidneys of DKD rats restored after treatment with DHI. The expression of p38MAPK was activated by DHI.

Peroxisome proliferator-activated receptor gamma $(\operatorname{PPAR} \gamma)$ belongs to the ligand-activated type II nuclear receptor superfamily and predominantly expressed in adipose tissues [24]. In the kidney, PPAR $\gamma$ is mainly located in the medullary collecting duct $[25,26]$, and glomeruli and proximal tubules were also expressed in small amounts [25]. Activated PPAR $\gamma$ delayed the progress of DKD by improving insulin resistance [27], lowering blood pressure [28], ameliorating inflammation [29], reversing cell cycle arrest [30], increasing adiponectin [31], improving oxidative stress [32], and other mechanisms. On the other hand, the study has shown that PPAR $\gamma$ single nucleotide polymorphism (SNP) is 


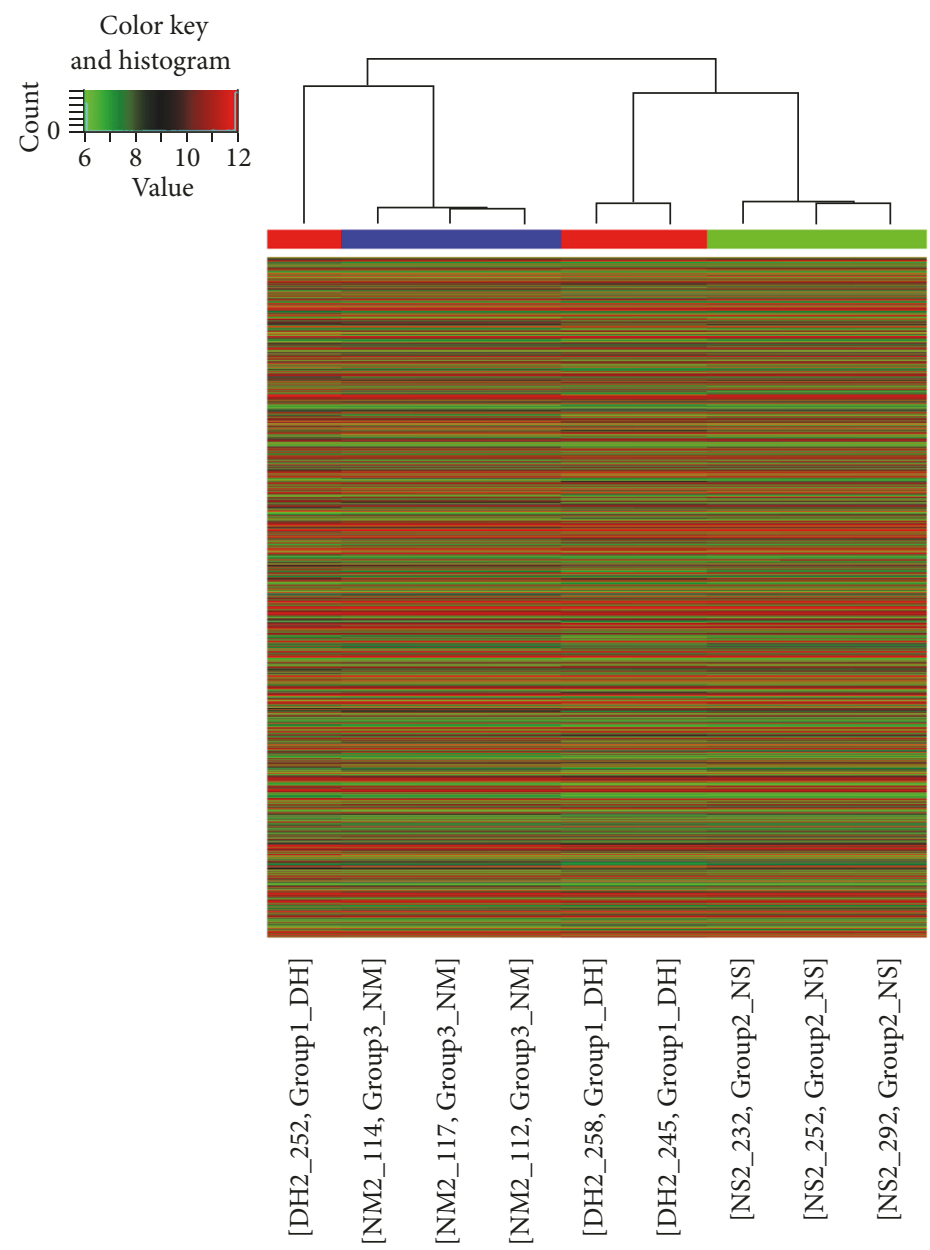

(a)

Sig pathway of DE gene

PPAR signaling pathway, Rattus norvegicus (rat)

Hypertrophic cardiomyopathy (HCM), Rattus norvegicus (rat)

Adipocytokine signaling pathway, Rattus norvegicus (rat)

Tyrosine metabolism, Rattus norvegicus (rat)

Dilated cardiomyopathy, Rattus norvegicus (rat)

Adrenergic signaling in cardiomyocytes, Rattus norvegicus (rat)

Proximal tubule bicarbonate reclamation, Rattus norvegicus (rat)

Renin-angiotensin system, Rattus norvegicus (rat)

Pertussis, Rattus norvegicus (rat)

Complement and coagulation cascades, Rattus norvegicus (rat)
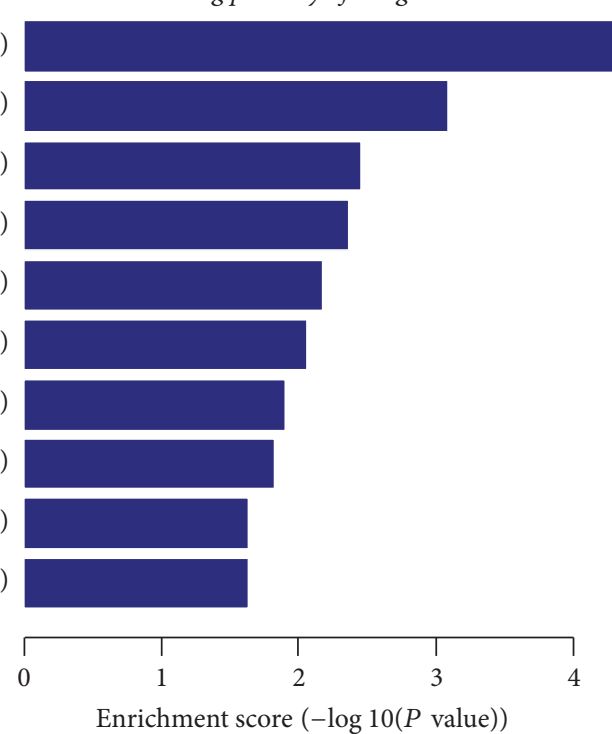

(b)

FIGURE 4: Cluster analysis and KEGG pathway enrichment analysis of differentially expressed genes. NM, NS, and DH groups, respectively, are represented by 3 samples which represent biologically independent duplicates. Red, a minimum twofold increase in expression; green, a minimum twofold reduction in expression. 

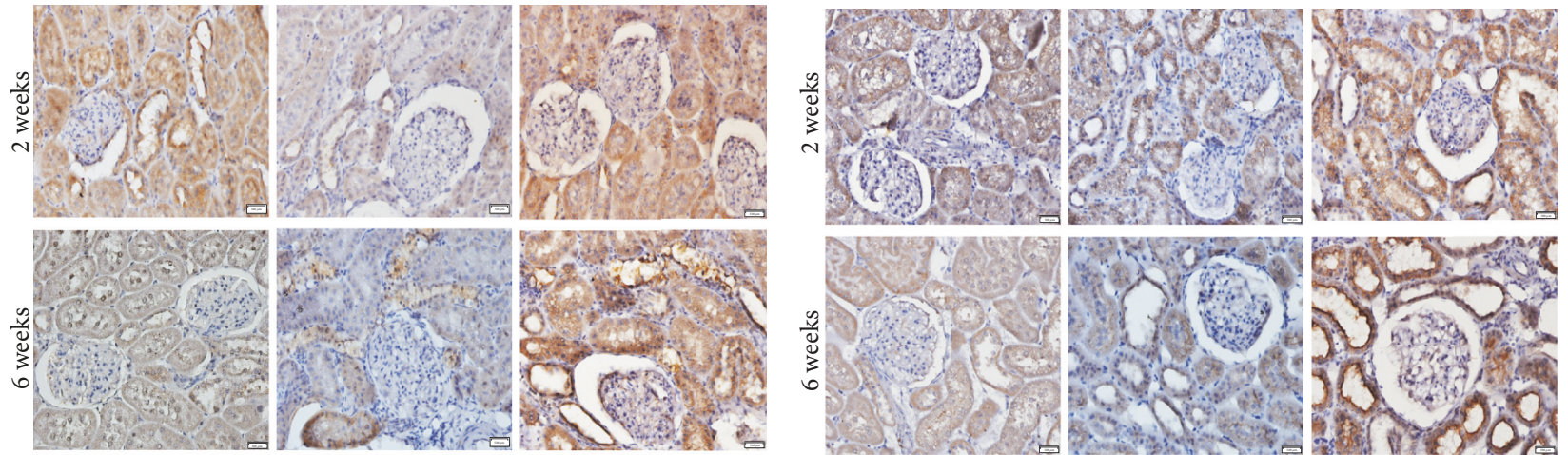

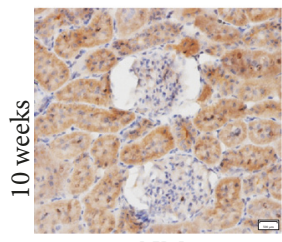

NM

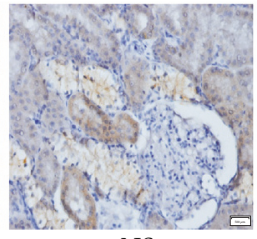

N

(a)

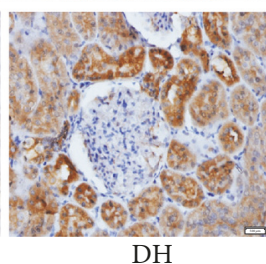

DH

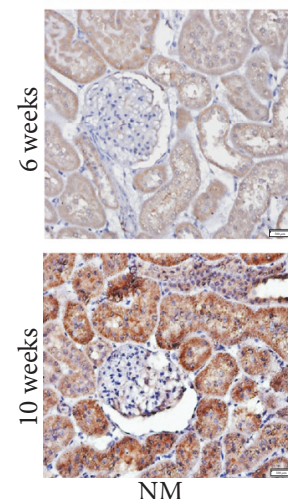

NM
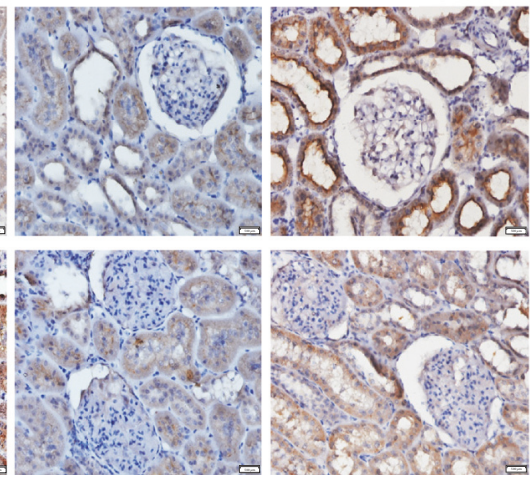

(b)

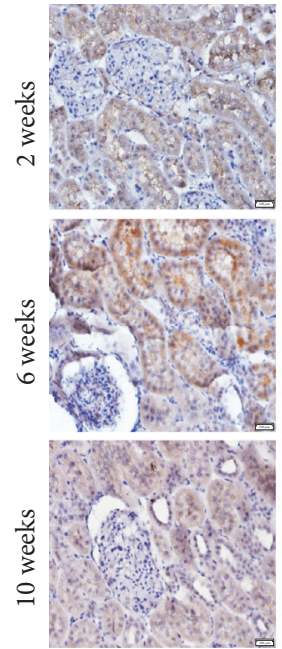

NM

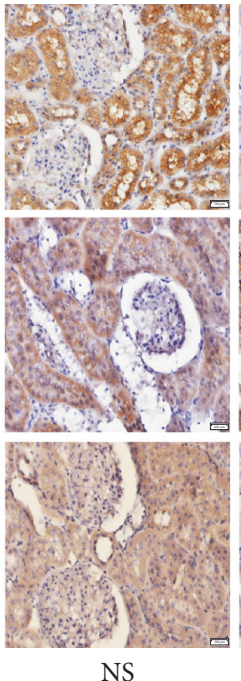

NS

(c)

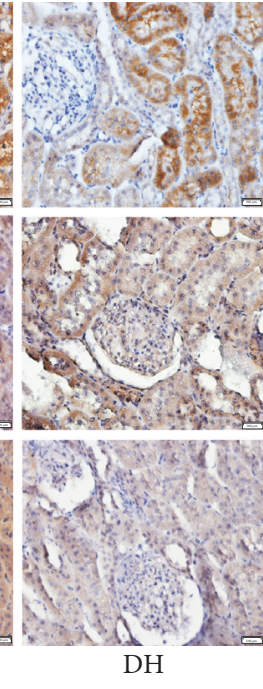

Figure 5: Effects of DHI on the expression of PPAR $\gamma$, UCP-1, and p38MAPK in the kidney. After the rats were sacrificed at the 2nd, 6th, and 10th week, the kidney $5 \mu \mathrm{m}$ cross paraffin sections were prepared and used to determine the expression of PPAR $\gamma$ (a), UCP-1 (b), and P38MAPK (c) by immunohistochemistry (scale bar: $500 \mu \mathrm{m}$ ).

associated with the risk of diabetic kidney disease [33]. All of these indicated that PPAR $\gamma$ signal pathway plays a protective role in DKD. The previous reports revealed that Danhong injection [34] and salvianolic acid B (one of the active ingredients of Danhong injection) [35] could suppress the maturation of dendritic cells through activating PPAR $\gamma$ and thus is mediated in the therapy of metabolic and inflammatory diseases, like diabetic kidney disease. In our study, the PPAR signaling pathway was upregulated in $\mathrm{DH}$ group. At the same time, immunohistochemistry staining suggested that the expression of PPAR $\gamma$ in rat renal tubules in DH group was higher than that in the NS group. It suggested that its renal protective effect was at least partially mediated by activation of $\operatorname{PPAR} \gamma$ signaling pathway.
In addition to the presence of glucose metabolism disorder, DKD patients also often accompany lipid metabolism disorder. An epidemiological survey showed that low HDL$\mathrm{C}$ and high TG levels were independent risk factors for the development and progression of renal disease in type 2 diabetic outpatients [36]. In contrast, lowering lipid levels can protect kidney function. Yokoyama et al. found that polyunsaturated fatty acid diet can reduce the lipid deposition in the kidneys and delay the progress of DKD [37]. It is reported that DHI treatment with hyperlipidemia rats was accompanied by significantly increased lipolysis in the liver and decreased fatty acid synthesis [38]. Moreover, agonist of PPAR $\gamma$ increased liver $\mathrm{X}$ receptor gene expression that mediated cholesterol efflux in glomerular mesangial cells [39] 
and thus reduced intracellular lipid accumulation [40]. In this study, we proved that DHI could decrease the level of serum lipids, especially cholesterol levels. So, DHI may be through $\operatorname{PPAR} \gamma$ signal pathway to reduce lipid levels to play its role in the protection of DKD rats.

Uncoupling protein-1 (UCP-1), which was enriched in the PPAR $\gamma$ signal pathway predicted by the KEGG pathway enrichment analysis, is a mitochondrial inner membrane protein and stimulates thermogenesis by uncoupling oxidative phosphorylation from the respiratory chain [41]. Through binding to PPAR $\gamma$ response elements in the UCP enhancer, referred to as UCP regulatory element 1 (URE1), PPAR $\gamma$ positively regulated the expression of UCP in HIB-1B cells [42]. Retinoid X receptor, ligand of PPAR $\gamma$, was also identified as a transactivating factor of the UCP gene promoter [43]. In addition, the newly found PPAR $\gamma$ agonist Fraglide- 1 could increase expression level of the uncoupling protein- (UCP-) 1 [44]. As a downstream target gene of PPAR $\gamma$, UCP-1 together with PPAR $\gamma$ played a key role in the energy balance $[45,46]$. In an obesity and hyperglycemia animal model, thiazolidinedione derived partial PPAR $\gamma$ agonist GQ-16 increased UCP1 protein expression in interscapular brown adipose tissue (BAT) and in epididymal and inguinal white adipose tissue (WAT) to induce WAT browning and treat obesity [45]. Mu et al. reported that the browning effect of ginsenoside $\mathrm{Rb} 1$ on 3T3-L1 adipocytes evidenced by increased expression of UCP-1 depends on the induction of PPAR $\gamma$ [46]. Moreover, studies indicated that UCP-1 overexpression could eliminate the production of hyperglycemia-induced reactive oxygen species (ROS) in cultured mouse glomerular mesangial cells [47], and upregulation of UCP-1 was beneficial for DKD rats [48]. In our study, we found that the protein expression levels of PPAR $\gamma$ and UCP-1 in the rat kidney were both enhanced after DHI treatment. Therefore, we speculated that Danhong injection reduces kidney damage in diabetic rats partially through the energy metabolism regulation mediated by PPAR $\gamma / \mathrm{UCP}-1$ signaling pathway.

P38 mitogen-activated protein kinase (MAPK) is a member of the MAPK family activated by environmental stresses and inflammatory cytokines. It is reported that the number of interstitial p-p38MAPK-positive cells in patients and rats with DKD reflected the severity of interstitial lesions [49] and were associated with hyperglycemia, increased $\mathrm{HbA}(1) \mathrm{c}$ levels, albuminuria, and interstitial fibrosis [50]. Conversely, inhibition of the p38MAPK pathway had a beneficial effect of DKD [51]. Several studies have shown that PPAR $\gamma$ agonists exerted therapeutic effects on various diseases by inhibiting the activity of p38MAPK [52-54]. In diabetic kidney disease, the reduced cell proliferation and fibronectin expression [55] or decreased oxidative stress and renal fibrosis [56] were associated with expression changes of PPAR $\gamma$ and $\mathrm{p} 38 \mathrm{MAPK}$. In addition, Chang et al. reported that rosiglitazone could prevent AGE-induced iNOS expression by interfering with p38MAPK activity in cultured glomerular mesangial cells [57]. In a cardiac hypertrophy model in vitro, DHI downregulated the phosphorylation of p38MAPK [58]. Our results showed that DHI increased the expression of PPAR $\gamma$ and decreased the expression of p38MAPK. Therefore, we speculated that Danhong injection delays the development of diabetic kidney disease partially through the $\mathrm{p} 38 \mathrm{MAPK} / \mathrm{PPAR} \gamma$ signaling pathway. This effect may be related to the inflammation regulation of P38MAPK/PPAR $\gamma$ signaling pathway, and we need further studies to confirm it.

\section{Conclusion}

Danhong injection could delay the progress of diabetic kidney disease, and the effect might be achieved in part by activating the PPAR $\gamma$ signaling pathway. We should explore the detailed mechanism for DHI protection against DKD in future studies.

\section{Conflicts of Interest}

The authors declare no competing financial interests.

\section{Authors' Contributions}

Xue Yang and Xiang Xiao contributed equally to this work.

\section{Acknowledgments}

This study was supported in part by Youth Science and Technology Creative Research Groups of Sichuan Province (2015TD0013).

\section{References}

[1] W. Yang, J. Lu, J. Weng et al., "Prevalence of diabetes among men and women in China," The New England Journal of Medicine, vol. 362, no. 12, pp. 1090-1101, 2010.

[2] Y. Xu, L. Wang, J. He et al., "Prevalence and control of diabetes in Chinese adults," Journal of the American Medical Association, vol. 310, no. 9, pp. 948-958, 2013.

[3] I. H. de Boer, T. C. Rue, Y. N. Hall, P. J. Heagerty, N. S. Weiss, and J. Himmelfarb, "Temporal trends in the prevalence of diabetic kidney disease in the United States," Journal of the American Medical Association, vol. 305, no. 24, pp. 2532-2539, 2011.

[4] H.-H. Parving, B. M. Brenner, J. J. V. McMurray et al., "Cardiorenal end points in a trial of aliskiren for type 2 diabetes," The New England Journal of Medicine, vol. 367, no. 23, pp. 22042213, 2012.

[5] D. de Zeeuw, T. Akizawa, P. Audhya et al., "Bardoxolone methyl in type 2 diabetes and stage 4 chronic kidney disease," The New England Journal of Medicine, vol. 369, no. 26, pp. 2492-2503, 2013.

[6] R. Saran, B. Robinson, K. C. Abbott et al., "US renal data system 2016 annual data report: epidemiology of kidney disease in the United States," American Journal Of Kidney Diseases, vol. 69, no. 3, pp. A7-A8, 2017.

[7] L. Zhang, J. Long, W. Jiang et al., "Trends in chronic kidney disease in China," The New England Journal of Medicine, vol. 375, no. 9, pp. 905-906, 2016.

[8] Y.-Y. Xie, X. Xiao, J.-M. Luo et al., "Integrating qualitative and quantitative characterization of traditional Chinese medicine injection by high-performance liquid chromatography with diode array detection and tandem mass spectrometry," Journal of Separation Science, vol. 37, no. 12, pp. 1438-1447, 2014. 
[9] X. Liu, Z. Z. Wu, K. Yang, H. Y. Ding, and Y. J. Wu, "Quantitative analysis combined with chromatographic fingerprint for comprehensive evaluation of Danhong injection using HPLCDAD," Journal of Pharmaceutical and Biomedical Analysis, vol. 76, pp. 70-74, 2013.

[10] x. Jing, "Clinical study of fosinopril combined with danhong injection in treating early diabetic nephropathy," Clinical Medicine, vol. 34, no. 4, pp. 50-51, 2014.

[11] H. Z. Li and W. Y. Limin, "Improvement of proteinuria in patients with type 2 diabetic nephropathy by Danhong injection," journal of China Prescription Drug, vol. 14, no. 6, pp. 75-76, 2016.

[12] S. K. Kim, K.-H. Jung, and B.-C. Lee, "Protective effect of tanshinone IIA on the early stage of experimental diabetic nephropathy," Biological \& Pharmaceutical Bulletin, vol. 32, no. 2, pp. 220-224, 2009.

[13] M. Liu, Q. Pan, Y. Chen et al., "Administration of Danhong Injection to diabetic $\mathrm{db} / \mathrm{db}$ mice inhibits the development of diabetic retinopathy and nephropathy," Scientific Reports, vol. 5, article 11219, 2015.

[14] Y. He, H. Wan, Y. Du et al., "Protective effect of Danhong injection on cerebral ischemia-reperfusion injury in rats," Journal of Ethnopharmacology, vol. 144, no. 2, pp. 387-394, 2012.

[15] M. Liu, Y. Chen, X. Yang et al., "DanHong Injection inhibits the development of primary abdominal aortic aneurysms in apoE knockout mice," Chinese Science Bulletin, vol. 59, no. 13, pp. 1366-1373, 2014.

[16] Y. Guan, Y. Yin, Y.-R. Zhu et al., "Dissection of mechanisms of a Chinese medicinal formula: danhong injection therapy for myocardial ischemia/reperfusion injury in vivo and in vitro," Evidence-Based Complementary and Alternative Medicine, vol. 2013, Article ID 972370, 12 pages, 2013.

[17] X.-J. Ma, S.-J. Yin, J.-C. Jin et al., "Synergistic protection of danhong injection and ischemic postconditioning on myocardial reperfusion injury in minipigs," Chinese Journal of Integrative Medicine, vol. 16, no. 6, pp. 531-536, 2010.

[18] Z. Wang, F. Song, J. Li et al., "PET demonstrates functional recovery after treatment by danhong injection in a rat model of cerebral ischemic-reperfusion injury," Evidence-Based Complementary and Alternative Medicine, vol. 2014, Article ID 430757, 9 pages, 2014.

[19] L.-N. Gao, Y.-L. Cui, Q.-S. Wang, and S.-X. Wang, "Amelioration of Danhong injection on the lipopolysaccharide-stimulated systemic acute inflammatory reaction via multi-target strategy," Journal of Ethnopharmacology, vol. 149, no. 3, pp. 772-782, 2013.

[20] L.-M. Wan, L. Tan, Z.-R. Wang et al., "Preventive and therapeutic effects of Danhong injection on lipopolysaccharide induced acute lung injury in mice," Journal of Ethnopharmacology, vol. 149, no. 1, pp. 352-359, 2013.

[21] Y. Wang, L.-N. Gao, Y.-L. Cui, and H.-L. Jiang, "Protective effect of danhong injection on acute hepatic failure induced by lipopolysaccharide and D-galactosamine in mice," EvidenceBased Complementary and Alternative Medicine, vol. 2014, Article ID 153902, 8 pages, 2014.

[22] M. Zhang, K. Cui, Y. Zhu, and Y. Duan, "Dan Hong injection for diabetic nephropathy: A systematic review," Chinese Journal of Evidence-Based Medicine, vol. 9, no. 10, pp. 1087-1093, 2009.

[23] S. L. Ren, "Meta - analysis of the Effect of Danhong Injection on Urinary Protein and Renal Function," Chinese Journal of Integrated Traditional and Western Nephrology, vol. 16, no. 9, pp. 825-828, 2015.
[24] A. Chawla, E. J. Schwarz, D. D. Dimaculangan, and M. A. Lazar, "Peroxisome proliferator-activated receptor (PPAR) $\gamma$ : adiposepredominant expression and induction early in adipocyte differentiation," Endocrinology, vol. 135, no. 2, pp. 798-800, 1994.

[25] T. Yang, D. E. Michele, J. Park et al., "Expression of peroxisomal proliferator-activated receptors and retinoid $X$ receptors in the kidney," American Journal of Physiology-Renal Physiology, vol. 277, no. 6, pp. F966-F973, 1999.

[26] Y. Guan, Y. Zhang, L. Davis, and M. D. Breyer, "Expression of peroxisome proliferator-activated receptors in urinary tract of rabbits and humans," American Journal of Physiology-Renal Physiology, vol. 273, no. 6, pp. F1013-F1022, 1997.

[27] S. Tiwari, V. K. M. Halagappa, S. Riazi, X. Hu, and C. A. Ecelbarger, "Reduced expression of insulin receptors in the kidneys of insulin-resistant rats," Journal of the American Society of Nephrology, vol. 18, no. 10, pp. 2661-2671, 2007.

[28] Q. N. Diep, M. E. Mabrouk, J. S. Cohn et al., "Structure, endothelial function, cell growth, and inflammation in blood vessels of angiotensin II-infused rats: role of peroxisome proliferatoractivated receptor- $\gamma$," Circulation, vol. 105, no. 19, pp. 22962302, 2002.

[29] S. Ohga, K. Shikata, K. Yozai et al., "Thiazolidinedione ameliorates renal injury in experimental diabetic rats through antiinflammatory effects mediated by inhibition of NF-kappaB activation," American Journal of Physiology-Renal Physiology, vol. 292, no. 4, pp. F1141-F1150, 2007.

[30] T. Okada, J. Wada, K. Hida et al., “Thiazolidinediones ameliorate diabetic nephropathy via cell cycle-dependent mechanisms," Diabetes, vol. 55, no. 6, pp. 1666-1677, 2006.

[31] Y. Miyazaki, E. Cersosimo, C. Triplitt, and R. A. DeFronzo, "Rosiglitazone decreases albuminuria in type 2 diabetic patients," Kidney International, vol. 72, no. 11, pp. 1367-1373, 2007.

[32] A. Gumieniczek, "Effect of the new thiazolidinedionepioglitazone on the development of oxidative stress in liver and kidney of diabetic rabbits," Life Sciences, vol. 74, no. 5, pp. 553-562, 2003.

[33] H. Zhang, S. Zhu, and J. Chen, "Peroxisome proliferatoractivated receptor $\gamma$ polymorphism Prol2Ala is associated with nephropathy in type 2 diabetes: evidence from meta-analysis of 18 studies," Diabetes Care, vol. 35, no. 6, pp. 1388-1393, 2012.

[34] H. Liu, S. Wang, A. Sun et al., "Danhong inhibits oxidized low-density lipoprotein-induced immune maturation of dentritic cells via a peroxisome proliferator activated receptor $\gamma$ mediated pathway," Journal of Pharmacological Sciences, vol. 119, no. 1, pp. 1-9, 2012.

[35] A. Sun, H. Liu, and S. Wang, "Salvianolic acid B suppresses maturation of human monocyte-derived dendritic cells by activating PPAR $\gamma$," British Journal of Pharmacology, vol. 164, no. 8, pp. 2042-2053, 2011.

[36] G. T. Russo, S. De Cosmo, F. Viazzi et al., "Plasma triglycerides and HDL-C levels predict the development of diabetic kidney disease in subjects with type 2 diabetes: The AMD annals initiative," Diabetes Care, vol. 39, no. 12, pp. 2278-2287, 2016.

[37] M. Yokoyama, K. Tanigawa, T. Murata et al., "Dietary polyunsaturated fatty acids slow the progression of diabetic nephropathy in streptozotocin-induced diabetic rats," Nutrition Research, vol. 30, no. 3, pp. 217-225, 2010.

[38] J. Chen, J. Deng, Y. Zhang et al., "Lipid-lowering effects of Danhong injection on hyperlipidemia rats," Journal of Ethnopharmacology, vol. 154, no. 2, pp. 437-442, 2014.

[39] J. Wu, Y. Zhang, N. Wang et al., "Liver X receptor- $\alpha$ mediates cholesterol efflux in glomerular mesangial cells," American 
Journal of Physiology-Renal Physiology, vol. 287, no. 5, pp. F886F895, 2004.

[40] X. Z. Ruan, J. F. Moorhead, R. Fernando, D. C. Wheeler, S. H. Powis, and Z. Varghese, "PPAR agonists protect mesangial cells from interleukin $1 \beta$-induced intracellular lipid accumulation by activating the ABCA1 cholesterol efflux pathway," Journal of the American Society of Nephrology, vol. 14, no. 3, pp. 593-600, 2003.

[41] D. G. Nicholls and R. M. Locke, "Thermogenic mechanisms in brown fat," Physiological Reviews, vol. 64, no. 1, pp. 1-64, 1984.

[42] I. B. Sears, M. A. MacGinnitie, L. G. Kovacs, and R. A. Graves, "Differentiation-dependent expression of the brown adipocyte uncoupling protein gene: regulation by peroxisome proliferator-activated receptor gamma," Molecular and Cellular Biology, vol. 16, no. 7, pp. 3410-3419, 1996.

[43] A.-M. Cassard-Doulcier, M. Larose, J. C. Matamala, O. Champigny, F. Bouillaud, and D. Ricquier, "In vitro interactions between nuclear proteins and uncoupling protein gene promoter reveal several putative transactivating factors including Ets1, retinoid $\mathrm{X}$ receptor, thyroid hormone receptor, and a CACCC boxbinding protein," The Journal of Biological Chemistry, vol. 269, no. 39, pp. 24335-24342, 1994.

[44] Y. Tsujino, "A new agonist for peroxisome proliferation-activated receptor $\gamma(\operatorname{PPAR} \gamma)$, fraglide-1 from zhenjiang fragrant vinegar: Screening and characterization based on cell culture experiments," Journal of Oleo Science, vol. 66, no. 6, pp. 615-622, 2017.

[45] M. S. Coelho, C. L. De Lima, C. Royer et al., "GQ-16, a TZDderived partial PPAR $\gamma$ agonist, induces the expression of thermogenesis-related genes in brown fat and visceral white fat and decreases visceral adiposity in obese and hyperglycemic mice," PLoS ONE, vol. 11, no. 5, Article ID e0154310, 2016.

[46] Q. Mu, X. Fang, X. Li et al., "Ginsenoside Rb1 promotes browning through regulation of PPAR $\gamma$ in 3T3-L1 adipocytes," Biochemical and Biophysical Research Communications, vol. 466, no. 3, pp. 530-535, 2015.

[47] S. Kiritoshi, T. Nishikawa, K. Sonoda et al., "Reactive oxygen species from mitochondria induce cyclooxygenase-2 gene expression in human mesangial cells: potential role in diabetic nephropathy," Diabetes, vol. 52, no. 10, pp. 2570-2577, 2003.

[48] Y. Shen, M. Fukushima, Y. Ito et al., "Verification of the antidiabetic effects of cinnamon (Cinnamomum zeylanicum) using insulin-uncontrolled type 1 diabetic rats and cultured adipocytes," Bioscience, Biotechnology, and Biochemistry, vol. 74, no. 12, pp. 2418-2425, 2010.

[49] N. Sakai, T. Wada, K. Furuichi et al., "Involvement of extracellular signal-regulated kinase and p38 in human diabetic nephropathy," American Journal of Kidney Diseases, vol. 45, no. 1, pp. 54-65, 2005.

[50] L. Adhikary, F. Chow, D. J. Nikolic-Paterson et al., "Abnormal p38 mitogen-activated protein kinase signalling in human and experimental diabetic nephropathy," Diabetologia, vol. 47, no. 7, pp. 1210-1222, 2004.

[51] D.-S. Jung, J. J. Li, S.-J. Kwak et al., "FR167653 inhibits fibronectin expression and apoptosis in diabetic glomeruli and in high-glucose-stimulated mesangial cells," American Journal of Physiology-Renal Physiology, vol. 295, no. 2, pp. F595-F604, 2008.

[52] X. He, L. Feng, H. Meng, X. Wang, and S. Liu, "Rosiglitazone protects dopaminergic neurons against lipopolysaccharideinduced neurotoxicity through inhibition of microglia activation," International Journal of Neuroscience, vol. 122, no. 9, pp. 532-540, 2012.
[53] Y. Yang, L.-H. Zhao, B. Huang et al., "Pioglitazone, a PPAR $\gamma$ agonist, inhibits growth and invasion of human hepatocellular carcinoma via blockade of the rage signaling," Molecular Carcinogenesis, vol. 54, no. 12, pp. 1584-1595, 2015.

[54] S. Sharma, P. M. Sharma, D. S. Mistry et al., "PPARG regulates gonadotropin-releasing hormone signaling in LbetaT2 cells in vitro and pituitary gonadotroph function in vivo in mice," Biology of Reproduction, vol. 84, no. 3, pp. 466-475, 2011.

[55] X. Li, W. Liu, Q. Wang et al., "Emodin suppresses cell proliferation and fibronectin expression via p38MAPK pathway in rat mesangial cells cultured under high glucose," Molecular and Cellular Endocrinology, vol. 307, no. 1-2, pp. 157-162, 2009.

[56] A. P. Lakshmanan, K. Watanabe, and R. A. Thandavarayan, "Telmisartan attenuates oxidative stress and renal fibrosis in streptozotocin induced diabetic mice with the alteration of angiotensin-(1-7) mas receptor expression associated with its PPAR- $\gamma$ agonist action," Free Radical Research, vol. 45, no. 5, pp. 575-584, 2011.

[57] P.-C. Chang, T.-H. Chen, C.-J. Chang, C.-C. Hou, P. Chan, and H.-M. Lee, "Advanced glycosylation end products induce inducible nitric oxide synthase (iNOS) expression via a p38 MAPKdependent pathway," Kidney International, vol. 65, no. 5, pp. 1664-1675, 2004.

[58] H.-P. Mao, X.-Y. Wang, Y.-H. Gao et al., "Danhong injection attenuates isoproterenol-induced cardiac hypertrophy by regulating p38 and NF- $\kappa$ b pathway," Journal of Ethnopharmacology, vol. 186, pp. 20-29, 2016. 


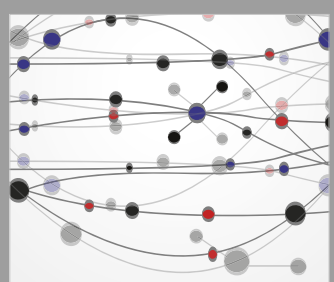

The Scientific World Journal
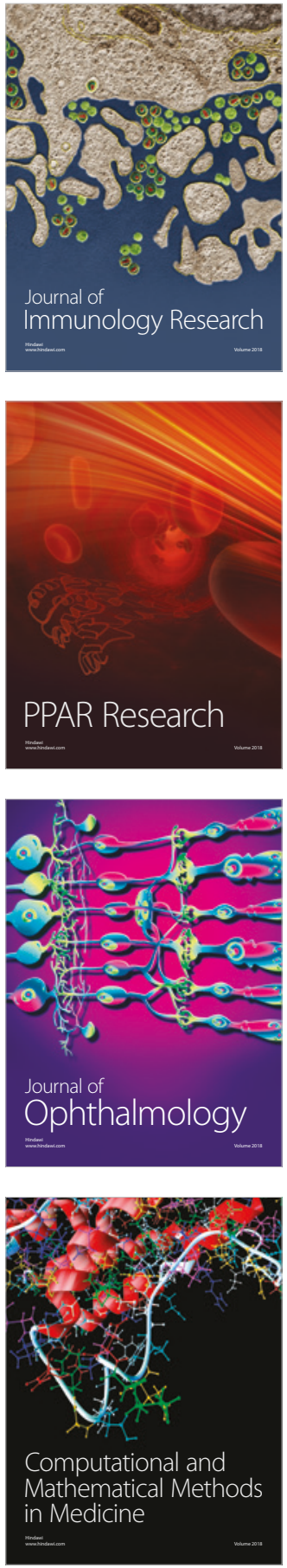

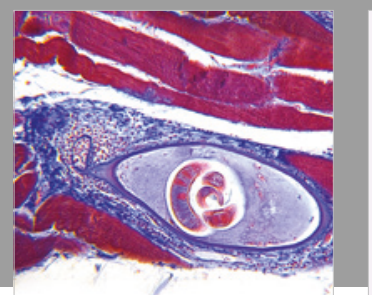

Gastroenterology Research and Practice

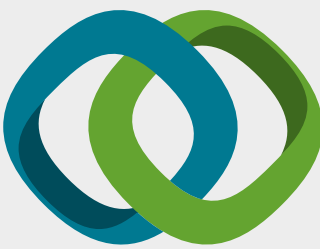

\section{Hindawi}

Submit your manuscripts at

www.hindawi.com
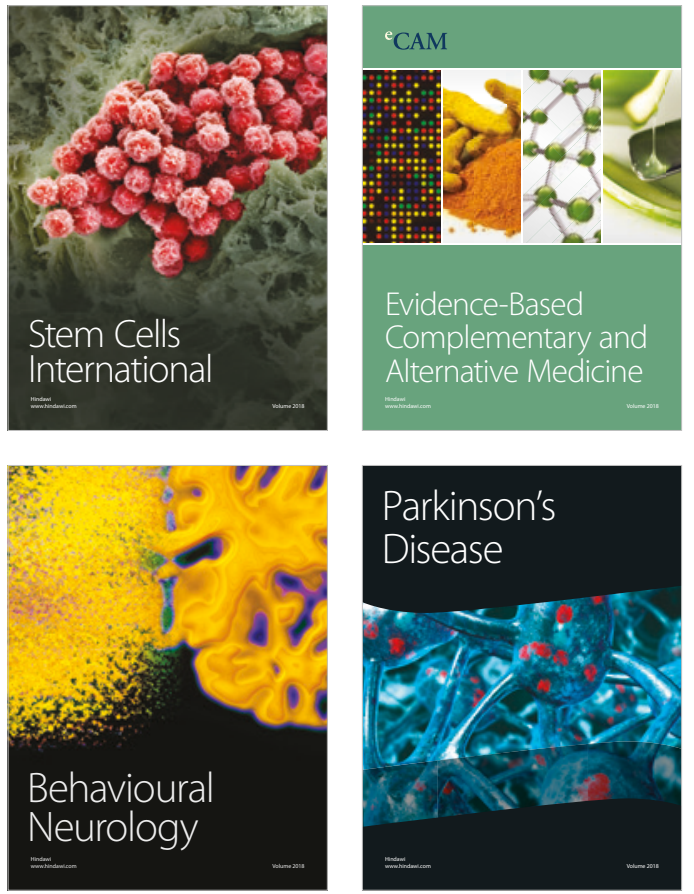

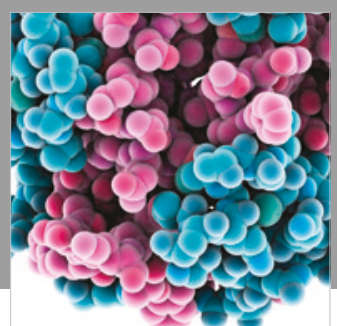

ournal of

Diabetes Research

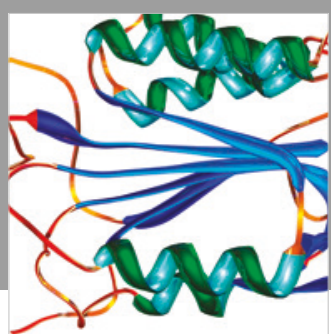

Disease Markers
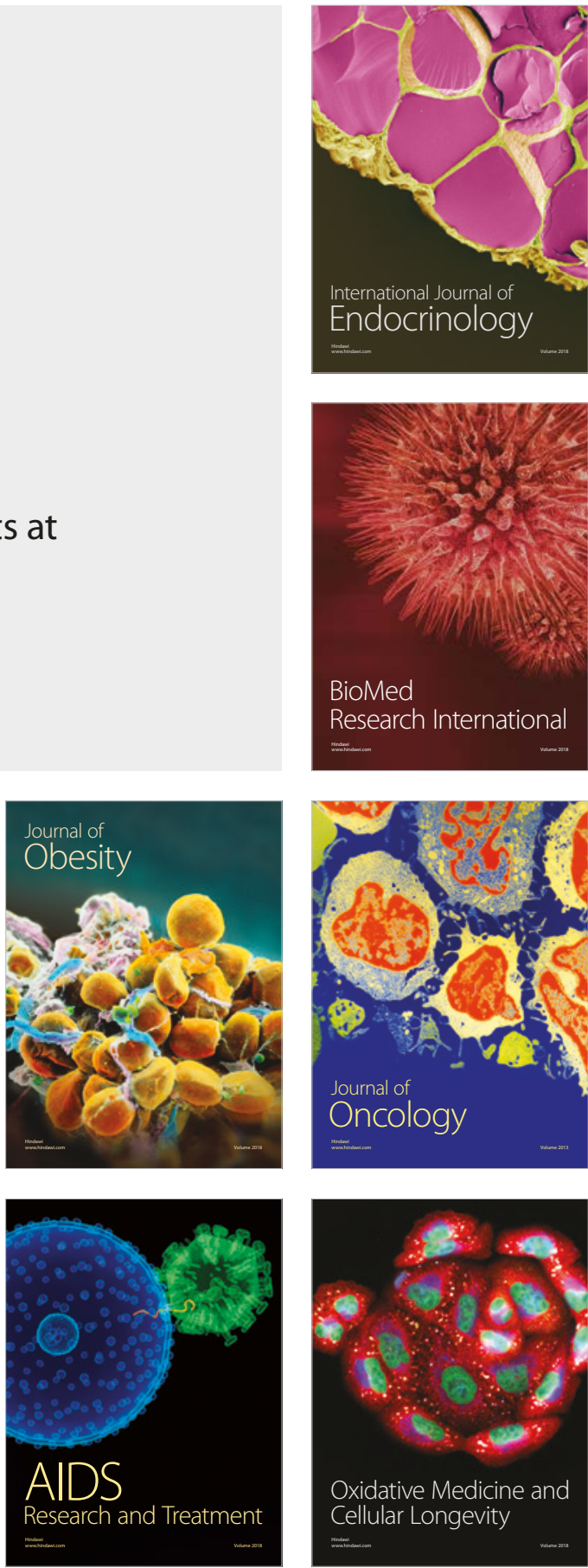